\title{
One Parameter Selection Method of the BP Neural Network on Real Options Pricing
}

\author{
Xiao Lan \\ School of Economics and Management \\ Wuhan Institute of Shipbuilding Technology \\ Wuhan, China \\ e-mail: xiannie@126.com
}

\begin{abstract}
Based on the theory of BP neural network, the evaluation prediction model of real options pricing was established. Some quantized and non-quantized factors were all considered into the model such as evaluation value uncertainty, investment cost, investment opportunity duration, share degree, environment risk and so on. With one given example, the input parameters of the model were optimized by means of genetic algorithm and the detailed input parameters optimization process was also pointed out by dint of one calculation flow chart. The results show that the method not only guarantees the precision of calculation, but also reduces the number of input variables in the prediction model. At the same time, it can avoid that the calculation does not converge because of lots of input parameters to the prediction model. In addition, this can also reduce the subjectivity of the Black-Scholes model and make the evaluation of real options pricing more accurate. As a result, one good prediction effect has been obtained again.
\end{abstract}

Keywords- Real options pricing; BP neural network; Genetic algorithm; Parameters optimization;Prediction

\section{INTRODUCTION}

The evaluation process of real options pricing is very complicated and various factors involved in the assessment determine the characteristics of real options pricing .Because of the strong nonlinear relationship between these factors, it is very difficult to give one accurate or specific mathematical formula to predict the assessment of real options pricing. The characteristics of physical assets were defined with the method of twin security in [1], but it was very difficult to define the value of real options with determined parameters in view of the different situations. Some characteristics of special assets were pointed out that they were very difficult to be mathematically and clearly assessed, and some mathematical equation descriptions had their own shortcoming in [2]. A hybrid wavelet neural network based on the Black-Scholes model was proposed for the case study on Hong Kong derivative market in [3]. The strategic growth options under uncertainty was analyzed and imperfect competition situation and pointed out that the important risk factors of volatility was not the impeding investment in [4]. The risk of option pricing caused by the kind of discontinuous hedging was analyzed and the ratio of risk of several options in American stock market was given in [5]. The shortcomings of the existing research the corresponding option pricing based on neural network was described in [6]. In addition, based on BlackScholes model, one radial basis function(RBF) neural network prediction model of the warrant pricing was pointed out with the given practice example in [7]. In fact, with the help of the training sample data and self learning function, BP artificial neural network algorithm(BP) can realize the nonlinear mapping relationship among the different dimension spaces. At the same time, it has better applicability to solve the evaluation and prediction of the real options pricing.

However, during the process of prediction of the real options pricing by the way of $\mathrm{BP}$, there may be trapped in local minima problem because of its slow convergence speed or immoderate prediction model parameters ${ }^{[8]}$. All these will affect the precision of BP prediction. Therefore, it is very important how to select related input parameters in the BP prediction model for better results. In this article, aiming at one specific evaluation example of the company, the prediction of the real options pricing by the way of BP which input parameters were optimized by the genetic algorithm(GA) was carried out and had one good prediction effect.

\section{BP EVALUATION MODEL OF REAL OPTIONS PRICING}

\section{A. Influence factor selection}

The evaluation process of real options pricing can be achieved by building one nonlinear mapping relationship between the influence factors and final evaluation value Therefore, the final evaluation results of real options pricing will be directly determined by the influence performance of the input parameters in the BP evaluation model. In this paper, according to the related research, the input factors will be identified as two categories. Among them, one kind is the influence factors which can be quantified, another kind is the influence factors which can't be easy to be quantified. The former mainly includes: evaluation value uncertainty $(\sigma)$, investment $\operatorname{cost}(K)$, investment opportunity duration $(T)$, risk-free interest rate of project operation $\left(R_{f}\right)$ and expected present value of cash flow $(S)$ during the project evaluation. Thinking of some factors such as non-transaction, share degree, pre-empt degree, composite and environmental risk of real options, the latter will involve share $\operatorname{degree}(S D)$, pre-empt degree $(P D)$, competitor strategy $(C S)$, management $\operatorname{risk}(M R)$ and environment $\operatorname{risk}(E R)$ into the analysis model 
of the evaluation of real options pricing ${ }^{[9]}$. It can help perfectly reflect the essence of the evaluation of real options pricing. According to the influence degree of these factors of real options pricing, they will be divided into five levels, and every levels will be defined by the given numbers, as shown in table I .

According to the different change trend in a period of time, one incremental development value will be numerically defined in order to reflect some tiny change trend as shown in table II.

TABLE I. NUMERICAL DEFINITION OF THE NON-QUANTIFIED INFLUENCE FACTORS

\begin{tabular}{|c|c|c|c|c|c|}
\hline \multirow{2}{*}{$\begin{array}{c}\text { Influence } \\
\text { Degree }\end{array}$} & $\begin{array}{c}\text { Important } \\
\text { influence }\end{array}$ & $\begin{array}{c}\text { Great infl } \\
\text { uence }\end{array}$ & $\begin{array}{c}\text { Greater } \\
\text { influence }\end{array}$ & $\begin{array}{c}\text { Less } \\
\text { influence }\end{array}$ & $\begin{array}{c}\text { Tiny } \\
\text { influenc } \\
\text { ence }\end{array}$ \\
\hline $\begin{array}{c}\text { Definitio } \\
\mathrm{n}\end{array}$ & 1 & 0.8 & 0.6 & 0.4 & 0.2 \\
\hline
\end{tabular}

TABLE II. ADDITIONALLY NUMERICAL DEFINITION OF LOCAL CHANGE TREND

\begin{tabular}{|c|c|c|c|}
\hline \multirow{2}{*}{$\begin{array}{c}\text { Change } \\
\text { Trend }\end{array}$} & \multicolumn{3}{|c|}{ Change Trend Classification } \\
\cline { 2 - 4 } & Stable development & Great change & Decisive change \\
\hline $\begin{array}{c}\text { Co-rotating } \\
\text { Development }\end{array}$ & 0.01 & 0.02 & 1 \\
\hline $\begin{array}{c}\text { Reverse } \\
\text { Development }\end{array}$ & -0.01 & -0.02 & -1 \\
\hline
\end{tabular}

B. BP model by means of GA optimization parameters selection

1) Determining the structure of BP prediction model

Based on the calculation rule of BP, the topology map of BP prediction of real options pricing is shown in Fig.1 according to nonlinear relationship between the input parameters and output value during the course of the evaluation. In Fig.1, one input parameters matrix $I_{\mathrm{n}}$ is composed of some parameters such as $S, K$ and $M R$ and so on. Connection matrix $U_{\mathrm{nh}}$ is composed of hidden layer parameter matrix and input layer parameter matrix. Connection matrix $V_{\mathrm{hm}}$ is composed of hidden layer parameter matrix and output layer parameter matrix. By means of operation and self-learning process of the connection matrix $U_{\mathrm{nh}}$ and $V_{\mathrm{hm}}$, the evaluation prediction value matrix $Y_{\mathrm{m}}$ of real options pricing will be gained. In the end, a nonlinear mapping relationship between input parameters and the evaluation prediction value $V$ of real options pricing will be set up.

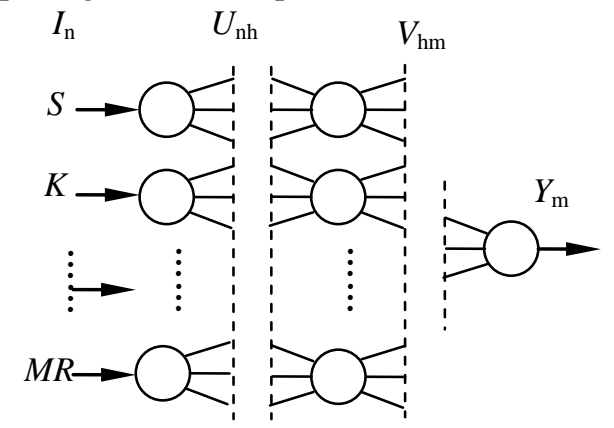

Figure 1. Topology map of BP nerual network.

\section{2) Genetic algorithm design}

Due to the complex rules of extraction algorithm which are proportional to the number of nodes and connection matrixes of BP neural network, the method of GA tournament selection is used to effectively remove the redundant nodes and branches from the weight matrix. At the same time, the best individual preservation method is used to select input variables and remove unnecessary nodes and branches. GA is operated as shown in Fig.2. Here, mutation probability is 0.1 and the selection range of given maximum genes is from 1 to 100 . When the number of the calculation iteration is the equal to the maximum of genes selection, the iteration algorithm will be stopped.

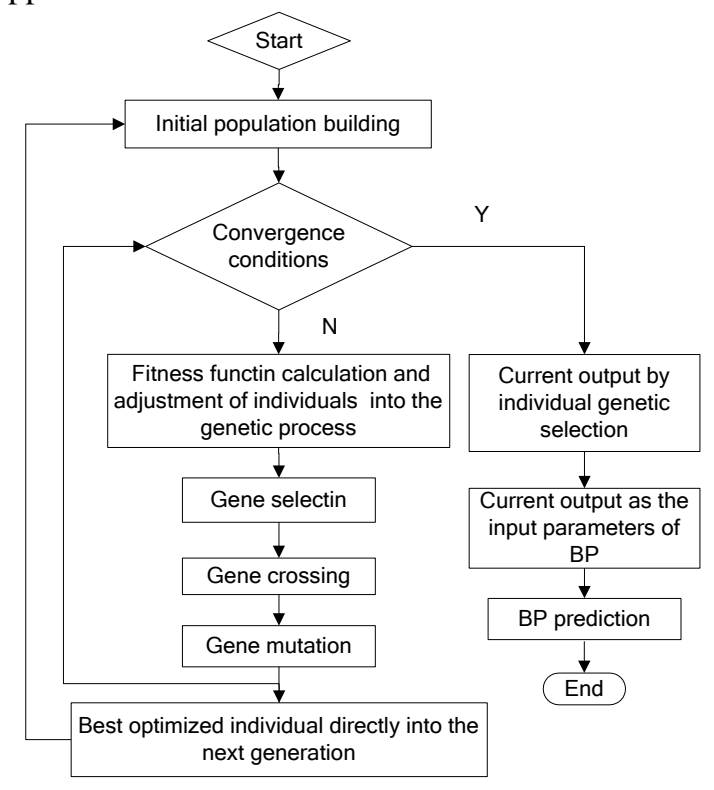

Figure 2. Calculation flow chart.

\section{3) Network variable selection optimization}

The degree of correlation among the value of input variables and hidden layer nodes and network output value may reflect the influence of input variables on the output value in BP prediction of real options pricing to a certain extent. So in order to analyze the influence which every input variable has an impact on the output value, some definition will be performed as following ${ }^{[10]}$.

$$
\left\{\begin{array}{l}
\alpha_{i j}=\left[\frac{u_{j i} w_{i j}}{\sigma\left(z_{i}\right)}\right]^{T} \\
\beta_{i}=\frac{v_{i \times 1} m}{\sigma(y)}
\end{array}\right.
$$

Where, $\alpha_{\mathrm{ij}}$ is the contribution of the input $\mathrm{j}$-th node on the $\mathrm{i}$-th node in hidden layer, $u_{\mathrm{ji}}$ is the matrix composed of the covariance between hidden layer node $z_{\mathrm{i}}$ and input variable $x_{\mathrm{j}}, w_{\mathrm{ij}}$ is the connection weight of input nodes and hidden layer nodes, $\beta_{\mathrm{i}}$ is the contribution of the $\mathrm{i}$-th node in hidden layer on the output value, $v_{\mathrm{i}}$ is the connection weight of the i-th node and output nodes in hidden layer, $m$ is the matrix composed of the $\mathrm{i}$-th hidden layer node output matrix $H_{\mathrm{i}}$ and $y$.

Optimal objective function is defined as following. 


$$
C_{j}=\sum_{i=1}^{q} \beta_{i} \alpha_{i j}
$$

Where, $C_{\mathrm{j}}$ is the contribution to overall output caused by i-th input variable, $q$ is the number of the nodes in hidden layer.

The fitness function in GA which is used to obtain the optimal solution is as following.

$$
\gamma=\sum_{j=1}^{m}\left\|C_{j} / \operatorname{sum}\left(C_{j}\right)\right\|
$$

Where, $\gamma$ is the fitness function in $\mathrm{GA}, \operatorname{sum}\left(C_{\mathrm{j}}\right)$ is the sum of all the elements in the matrix. $C_{\mathrm{j}}$ is the sum of the each line element in the matrix.

During the actual variable selection process, there are $P$ variables. If calculated contribution sum of input variables which number is $M(M<P)$ is close to 1 , these variables will be select to as important variables and other variables with relatively little contribution will be remove. By means of the above method, one new subset of variables will be obtained which only includes all the important input variables in the analysis model and will be trained by BP again. During the course, if the classification accuracy of the result achieved by trained BP doesn't reach the pre-determined standard, further net work pruning will be stopped and the neural network weights are restored to the last time set before the pruning. Otherwise, variables with relatively little contribution will continue to be removed. This process will be repeated until no variables can be eliminated. So far, the remaining variables can be thought to have an important influence on the prediction output value.

\section{SPECIFIC EXAMPLES}

One enterprise needs starting fund 4,000,000 dollars for the early products development. Two years later, the uncertainty of the marker risk and technology risk of the product development will be decreased. At term, $12,000,000$ dollars will be additionally invested and annual sales of $6,000,000$ dollars will be expected to realize. According to the historical data of some similar successful enterprises, the volatility of the forecasting data in the future is 0.4 and marker risk-free rate is $5 \%$. In accordance with the idea of real options, 4,000,000 dollars is currently invested, but follow-up 12,000,000 dollars investment and 6,000,000 dollars cash flow rights will be obtained two years later. The ratio of value and sale (sale/cash inflows) is 3.66 in some similar successful enterprises. Therefore, in the company, market opportunity brings into 22,000,000 dollars cash flow. So the sample input data which is used to evaluate the value of real options pricing can be given as following: the value of $K$ is $12,000,000$ dollars, the value of $S$ is $14,460,000$ dollars $(22,000,000$ dollars present value $)$, the value of $\sigma$ is 0.4 , the value of $T$ is 2 years and the value of $R_{\mathrm{f}}$ is 0.05 . According to the similar calculation method thinking of real options, the corresponding sample data can be obtained as shown in table III and tableIV.

According to (4), the sample data in table III and table IV can be normalized. All these normalized sample data will be used as the input parameters in BP model. In order to clearly describe the method, the former 10 sample data will be used as input parameters of the study sample in BP model and the eleventh sample data will be used for the test of the prediction effect.

$$
x_{i}^{\prime}=\frac{x_{i}-\min (x)}{\max (x)-\min (x)} \quad i=1,2,3, \ldots . n
$$

TABLE III. SAMPLE DATA

\begin{tabular}{|c|c|c|c|c|c|}
\hline \multirow{2}{*}{$\begin{array}{c}\text { Numbe } \\
\text { r }\end{array}$} & \multicolumn{5}{|c|}{ Input Parameters } \\
\cline { 2 - 6 } & $S$ (dollars $)$ & K (dollars $)$ & $\sigma$ & $T($ years $)$ & $R_{\mathrm{f}}$ \\
\hline 1 & 6000500 & 4500500 & 0.15 & 4 & 0.15 \\
\hline 3 & 2250000 & 2050000 & 0.3 & 5 & 0.05 \\
\hline 4 & 5150000 & 4250000 & 0.3 & 5 & 0.05 \\
\hline 5 & 7515900 & 75191000 & 0.4 & 6 & 0.06 \\
\hline 6 & 14462300 & 12550000 & 0.4 & 2 & 0.05 \\
\hline 7 & 2530000 & 1040000 & 0.25 & 1 & 0.2 \\
\hline 8 & 120000000 & 140000000 & 0.3 & 5 & 0.08 \\
\hline 9 & 119312000 & 110000000 & 0.2 & 2 & 0.1 \\
\hline 10 & 1679500 & 1450500 & 0.4 & 3 & 0.06 \\
\hline 11 & 1000000 & 1040000 & 0.2 & 1 & 0.08 \\
\hline
\end{tabular}

TABLE IV. SAMPLE DATA

\begin{tabular}{|c|c|c|c|c|c|}
\hline \multirow{2}{*}{\begin{tabular}{c} 
Numbe \\
\cline { 2 - 6 }
\end{tabular}} & \multicolumn{5}{|c|}{ Input Parameters } \\
\hline 1 & 0.21 & $P D$ & $C S$ & $E R$ & $M R$ \\
\hline 2 & 0.6 & 0.22 & 0.22 & 0.62 & 0.4 \\
\hline 3 & 0.82 & 0.41 & 0.2 & 0.6 & 0.8 \\
\hline 4 & 0.4 & 0.8 & 0.62 & 0.81 & 0.62 \\
\hline 5 & 0.21 & 1 & 0.8 & 0.2 & 0.61 \\
\hline 6 & 0.6 & 0.42 & 0.61 & 0.61 & 0.8 \\
\hline 7 & 0.8 & 0.6 & 0.4 & 0.2 & 0.41 \\
\hline 8 & 0.62 & 0.2 & 0.6 & 0.42 & 0.2 \\
\hline 9 & 0.41 & 0.81 & 0.61 & 0.4 & 0.82 \\
\hline 10 & 0.8 & 0.61 & 0.2 & 0.4 & 0.62 \\
\hline 11 & 0.41 & 0.42 & 0.6 & 0.6 & 0.41 \\
\hline
\end{tabular}

In the describing BP neural network training, neuron in the input layer is 10 and neuron in the output layer is 1. At the same time, levenberg-marquardt back propagation algorithm is used and tansig function is selected as transfer function between the input layer and hidden layer. On the other hand, purelin function is selected as transfer function between the hidden layer and output layer and trainlm-earngdm algorithm is also performed. Simultaneously, the mean square error function is used to calculate the error between the network output value and real value. When the training error is reduced to the specified range, GA is operated to variables selection according to (1)-(3), and then it can realize the 
optimization of the BP model ${ }^{[11]}$. The contribution proportion of every input variable on the output value is shown in Fig. 3.

As can be seen in Fig. 3, variable $T$ and variable $C S$ only account for 5 percent and 3 percent respectively, so it is very clear that their contribution on the output value is very tiny. In fact, the effect of variable $T$ on the value of real options pricing has been mainly reflected by variable $\mathrm{S}$ and variable $K$. On the other hand, the effect of variable $C S$ on the value of real options pricing has been mainly reflected by variable $S D$ and variable $M R$ and other variables. So in the evaluation of real options pricing, the two factors can not be considered. In addition, some variables such as $E R, P D, M R$ and $S D$ which are neglected in the current methods of real options pricing have great influence on the pricing results. Therefore, the above variables should be considered in the evaluation of real options pricing.

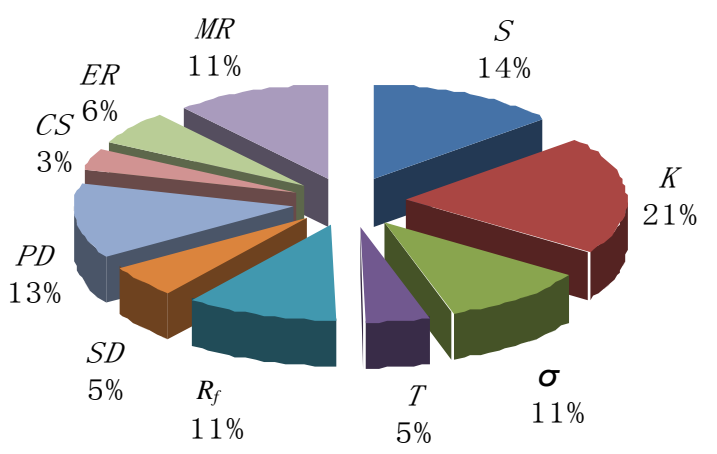

Figure 3. Contribution proportion of input variables on the output value.

Based on the above analysis, two variables called $T$ and $C S$ will be removed and a new subset of variables which is composed of the other eight variables will be established as the input parameters of the BP prediction model. Subsequently, the reconstructed BP model will be trained again. The training results are shown in Fig.4.

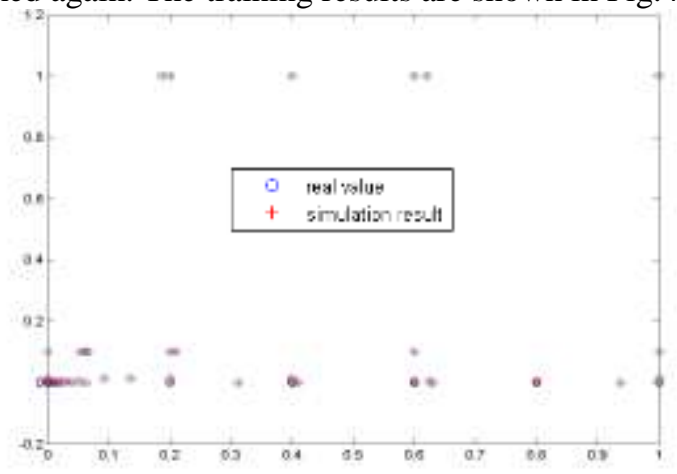

Figure 4. Training results of the reconstructed BP neural network.

As can be shown in Fig.4, the total mean squared error between the simulation value and goal value is about 3.9296e-021.Subsequently, the eleventh sample in Table 3 is tested in the reconstructed BP model and one verified value is obtained. As the result, the discrepancy between the verified value and real value is only 0.00003 . This denotes that the method of BP model by means of GA optimization parameters selection is distinctly effective.

The weights and thresholds are fixed in the optimized BP model and this can make the prediction network model better stability. In the end, one prediction model by means of the method of optimized BP model has been built for evaluation for real options pricing which is very stable and clipping and accurate. In practice, the prediction model should be adopted to the appraisal to the investment decision-making. As a result, better theoretical guidance will be gained for the investment decisionmaking.

\section{CONCLUSIONS}

Based on the above analysis, some conclusions can be obtained as following.

(1) In view of some shortcoming of the BP neural network such as slow convergence speed and local minimum solution and immoderate prediction model parameters, the BP model by means of GA optimization parameters selection in the evaluation prediction model of real options pricing can be used to avoid that the calculation does not converge. For some BP prediction problems which have too many input parameters, the method will have a good reference.

(2) In the evaluation prediction model of real options pricing, quantized factors and non-quantized factors are all considered for more accurate prediction. However, investment opportunity duration and competitor strategy have little contribution to the evaluation prediction, so they may not be considered in the prediction for reducing input parameters.

(3) By means of genetic algorithm, the input parameters of the model are optimized. As a result, the method not only guarantees the precision of calculation, but also reduces the number of input variables in the model. At the same time, it can also avoid inputting a lot of parameters to the prediction and reduce the subjectivity of the Black-Scholes model and make the evaluation of real options pricing more accurate.

\section{REFERENCES}

[1] Mao Ling. Study on Analysis Method of Real Options Evaluation. Journal of Nanjing University of Chemical Technology, 2000, vol. 2,pp.29-34.

[2] Martha Amram,Nalin Kulatilaka. Real Options. Beijing: Mechanical Industry Press, 2005, pp.153-194.

[3] Zhang Hongyan,Lin Hui,Jiang Cailou. Study on Option Pricing by Applying Hybrid Wavelet Networks and Genetic Algorithm.Journal of Systems Engineering,2010,vol.25,pp.43-49

[4] Amram M, Kulatilaka N. Real Options Managing Strategic Investment in an Uncertain World .Harvard Business School Press, 1999, pp.20-64.

[5] Xu Song. The Risk of Black-Scholes Option Pricing[J].Chinese Journal of Applied Probability and Statistics,vol.26,2010,pp:662671

[6] Xiao Lan.Research on Option Pricing Based on Neural Network,Securities \& Futures of China, 2011,vol.11,pp.6-7.

[7] Feng Demin,Bo Xiaoxu. The Warrant Prediction Model Research Based on Artificial Nerual Network. Market Economiy \& Price,2011,vol,5,pp.40-44

[8] Shang Jingqiang, Yuan Sicong, Wei Dongdong and Xiao Chang. Application of BP Neural Network and Genetic Algorithm in Fault Diagnosis of Tower Crane. Hoisting and Conveying Machinery, 2012, vol. 4,pp.61-64. 
[9] Li Fengying. Options Pricing Theory and Real Options Valuation.Statistics and Decision, 2001, vol. 6,pp.12-13.

[10] Ouyang Jianliang,Liu Lliming. Application of Integrative Improve BP Neural Network Algorithm in Stock Price Forecast,Computer\& Digital engineering,2011,vol.39 no.2, pp-57-60.

[11] Shi Feng, Wang Hui. MATLAB Intelligent Algorithm Analysis of 30 Cases . Beijing: Peking University of Aeronautics and Astronautics Press, 2011.
Author Induction:

Xiao Lan(1976-), female, master , majored in the study of the evaluation of real options pricing. 